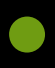 \\ hre a journal of mathematics
}

Betti numbers of order-preserving graph homomorphisms Lauren Guerra and Steven Klee mathematical sciences publishers 


\title{
Betti numbers of order-preserving graph homomorphisms
}

\author{
Lauren Guerra and Steven Klee
}

(Communicated by Jim Haglund)

\begin{abstract}
For graphs $G$ and $H$ with totally ordered vertex sets, a function mapping the vertex set of $G$ to the vertex set of $H$ is an order-preserving homomorphism from $G$ to $H$ if it is nondecreasing on the vertex set of $G$ and maps edges of $G$ to edges of $H$. In this paper, we study order-preserving homomorphisms whose target graph $H$ is the complete graph on $n$ vertices. By studying a family of graphs called nonnesting arc diagrams, we are able to count the number of orderpreserving homomorphisms (and more generally the number of order-preserving multihomomorphisms) mapping any fixed graph $G$ to the complete graph $K_{n}$.
\end{abstract}

\section{Introduction}

The study of graph homomorphisms has been the subject of a great deal of recent work in the fields of enumerative, algebraic, and topological combinatorics. The recent survey [Borgs et al. 2006] is an excellent source on the many facets of enumerating graph homomorphisms, while [Kozlov 2008] outlines a more topological approach. In this paper, we study combinatorial properties of order-preserving homomorphisms between two graphs $G$ and $H$ as introduced by Braun, Browder and Klee [Braun et al. 2011].

Throughout this paper, $V(G)$ and $E(G)$ will denote the vertex set and edge set respectively of a graph $G$. All graphs are assumed to be simple, meaning that loops and multiple edges are not allowed.

Let $G$ be a graph on vertex set $[m]=\{1,2, \ldots, m\}$ and let $H$ be a graph on vertex set $\left\{x_{1}, x_{2}, \ldots, x_{n}\right\}$. We order the vertex set of $G$ naturally, and we order the vertex set of $H$ by declaring that $x_{1}<x_{2}<\cdots<x_{n}$. An order-preserving homomorphism from $G$ to $H$ is a function $\varphi: V(G) \rightarrow V(H)$ such that

(1) if $1 \leq i<j \leq m$, then $\varphi(i) \leq \varphi(j)$, and

(2) if $(i, j) \in E(G)$, then $(\varphi(i), \varphi(j)) \in E(H)$.

MSC2010: primary 13D02; secondary 05A18, 06A06, 05C30.

Keywords: graph homomorphisms, Betti numbers, nonnesting partitions. 
An order-preserving homomorphism $\varphi: G \rightarrow H$ may be presented as a vector $[\varphi(i)]_{i=1}^{m}=[\varphi(1), \ldots, \varphi(m)]$.

Example 1.1. Let $G$ and $H$ be as follows:
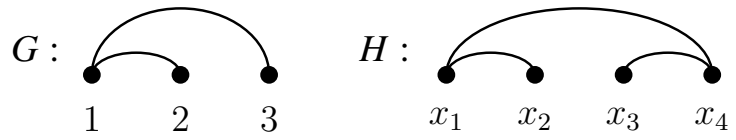

Define functions $\varphi_{1}, \varphi_{2}, \varphi_{3}: V(G) \rightarrow V(H)$ by

$$
\varphi_{1}:\left[x_{1}, x_{2}, x_{2}\right], \quad \varphi_{2}:\left[x_{1}, x_{2}, x_{4}\right], \quad \varphi_{3}:\left[x_{1}, x_{3}, x_{4}\right] .
$$

The functions $\varphi_{1}$ and $\varphi_{2}$ are order-preserving homomorphisms from $G$ to $H$. Notice that since $(2,3)$ is not an edge in $G$, having $\varphi_{1}(2)=\varphi_{1}(3)$ does not violate the definition of an order-preserving homomorphism. The function $\varphi_{3}$ is orderpreserving, but it is not a homomorphism since $(1,2) \in E(G)$, but $(\varphi(1), \varphi(2))=$ $\left(x_{1}, x_{3}\right) \notin E(H)$.

Rather than view each order-preserving homomorphism from $G$ to $H$ as a single function, it is often more convenient to encode several homomorphisms as a single object. An (order-preserving) multihomomorphism from $G$ to $H$ is a function $\eta: V(G) \rightarrow 2^{V(H)} \backslash \varnothing$ with the property that $[\varphi(i)]_{i=1}^{m}$ is an orderpreserving homomorphism from $G$ to $H$ for all possible choices of $\varphi(i) \in \eta(i)$ and $1 \leq i \leq m$. The complex of order-preserving homomorphisms from $G$ to $H$, denoted $\operatorname{OHOM}(G, H)$, is the collection of all multihomomorphisms from $G$ to $H$.

For any graphs $G$ and $H$, there is a geometric cell complex corresponding to $\operatorname{OHOM}(G, H)$ whose faces are labeled by multihomomorphisms from $G$ to $H$. While the geometry of $\operatorname{OHOM}(G, H)$ is very interesting in its own right, it is not the primary focus of this paper, and we will not spend any further time discussing it. For reasons that are motivated by this underlying geometry, we define the $d i$ mension of a multihomomorphism $\eta \in \operatorname{OHOM}(G, H)$ to be

$$
\operatorname{dim} \eta:=\sum_{i=1}^{m}(|\eta(i)|-1) .
$$

A zero-dimensional multihomomorphism is an order-preserving homomorphism. In this paper, we are primarily interested in a family of combinatorial invariants of $\operatorname{OHOM}(G, H)$ called its Betti numbers.

Definition 1.2. The $r$-th Betti number of the complex $\operatorname{OHOM}(G, H)$, denoted $\beta_{r}(G, H)$, counts the number of multihomomorphisms $\eta \in \operatorname{OHOM}(G, H)$ with $\operatorname{dim} \eta=r$. 
Example 1.3. Let $G$ and $H$ be as in Example 1.1. The following table encodes a one-dimensional multihomomorphism $\eta \in \operatorname{OHOM}(G, H)$ :

\begin{tabular}{c|c|c}
$\eta(1)$ & $\eta(2)$ & $\eta(3)$ \\
\hline$x_{1}$ & $x_{2}$ & $x_{2}$ \\
& & $x_{4}$
\end{tabular}

The two distinct choices of elements $[\varphi(1), \varphi(2), \varphi(3)]$ correspond to the orderpreserving homomorphisms $\varphi_{1}$ and $\varphi_{2}$ of Example 1.1.

The following proposition is a consequence of from our definitions of orderpreserving homomorphisms. We introduce the following notation, which will be used for the remainder of the paper. If $X$ and $Y$ are subsets of some totally ordered set (for our purposes, either $[m]$ or $\left\{x_{1}, \ldots, x_{n}\right\}$ ), we write $X \leq Y$ (or $X<Y$ ) to indicate that $x \leq y$ (similarly $x<y$ ) for all $x \in X$ and all $y \in Y$.

Proposition 1.4. Let $G$ and $H$ be graphs with

$$
V(G)=[m] \text { and } V(H)=\left\{x_{1}, \ldots, x_{n}\right\} .
$$

If $\eta \in \operatorname{OHOM}(G, H)$, then $\eta(1) \leq \eta(2) \leq \cdots \leq \eta(m)$. Moreover, if $(i, j)$ is an edge in $G$, then $\eta(i)<\eta(j)$.

The purpose of this paper is to determine the Betti numbers $\beta_{r}\left(G, K_{n}\right)$ of the complex of order-preserving homomorphisms between a fixed graph $G$ and the complete graph on $n$ vertices. In order to more easily compute the Betti numbers $\beta_{r}\left(G, K_{n}\right)$, we use the following series of reductions outlined in [Braun et al. 2011, Section 5]. All relevant definitions are deferred to Section 2.

(1) We show that for any graph $G$, there is a nonnesting partition $\mathscr{P}$ of $[m]$ and a corresponding graph $\Gamma_{\mathscr{P}}$ on $[\mathrm{m}]$, called an arc diagram, such that

$$
\operatorname{OHOM}\left(G, K_{n}\right)=\operatorname{OHOM}\left(\Gamma_{\mathscr{P}}, K_{n}\right) \text {. }
$$

(2) We define a weight function $\omega_{r}\left(\Gamma_{\mathscr{P}}, K_{n}\right)$ that counts the number of $r$-dimensional multihomomorphisms in $\operatorname{OHOM}\left(\Gamma_{\mathscr{P}}, K_{n}\right)$ "minimally" determined by $\mathscr{P}$. These weights are ultimately easier to compute than the Betti numbers of $\operatorname{OHOM}\left(\Gamma_{\mathscr{P}}, K_{n}\right)$.

(3) We define a partial order, denoted $\preceq$, on the family of nonnesting partitions of $[m]$ and show that

$$
\beta_{r}\left(\Gamma_{\mathscr{P}}, K_{n}\right)=\sum_{2 \preceq \mathscr{P}} \omega_{r}\left(\Gamma_{2}, K_{n}\right)
$$

In Section 3, we provide an explicit (and simple) closed formula for the weight function $\omega_{r}\left(\Gamma_{\mathscr{P}}, K_{n}\right)$ for any nonnesting partition $\mathscr{P}$. 


\section{Nonnesting partition graphs}

Nonnesting partitions. A partition $\mathscr{P}=\left\{P_{1}, \ldots, P_{t}\right\}$ of the set $[m]$ is a collection of nonempty subsets $P_{i} \subseteq[\mathrm{m}]$ (called blocks) such that $P_{i} \cap P_{j}=\varnothing$ for all $i \neq j$ and $P_{1} \cup \cdots \cup P_{t}=[m]$. We say that two blocks $P_{i}$ and $P_{j}$ nest if there exist $1 \leq a<b<c<d \leq m$ with $\{a, d\} \subseteq P_{i}$ and $\{b, c\} \subseteq P_{j}$ and there does not exist $e \in P_{i}$ with $b<e<c$. If no pair of blocks of $\mathscr{P}$ nest, we say that $\mathscr{P}$ is a nonnesting partition of $[\mathrm{m}]$. The family of nonnesting partitions was originally introduced and studied by Postnikov; see [Reiner 1997, Remark 2].

Example 2.1. The partition $\mathscr{P}_{1}=\{\{1,4\},\{2,5,6\},\{3\}\}$ of [6] is a nonnesting partition. The partition $\mathscr{P}_{2}=\{\{1,3,5\},\{2,6\},\{4\}\}$ is nesting since the blocks $\{1,3,5\}$ and $\{2,6\}$ nest.

It is more illuminating to represent a partition $\mathscr{P}$ of $[m]$ as a graph $\Gamma_{\mathscr{P}}$ as follows. Definition 2.2. Let $\mathscr{P}$ be a partition of $[m]$ and let $P_{i}=\left\{i_{1}, \ldots, i_{k}\right\}$ be a block of $\mathscr{P}$ with $i_{1}<\cdots<i_{k}$. The arc diagram $\Gamma_{\mathscr{P}}$ is the graph on vertex set $[m]$ whose edges are given by $\left(i_{j}, i_{j+1}\right)$ for consecutive elements of $P_{i}$ taken over all blocks of $\mathscr{P}$.

The name "arc diagram" is natural when the graph $\Gamma_{\mathscr{P}}$ is drawn so that its vertices are placed in a line and its edges are drawn as upper semicircular arcs, as shown in Example 2.3. In this representation, a partition $\mathscr{P}$ is nonnesting exactly when no arc of $\Gamma_{\mathscr{P}}$ is nested below another.

Example 2.3. Let $\mathscr{P}_{1}=\{\{1,4\},\{2,5,6\},\{3\}\}$ and $\mathscr{P}_{2}=\{\{1,3,5\},\{2,6\},\{4\}\}$ be the partitions of [6] discussed in Example 2.1. The arc diagrams $\Gamma_{\mathscr{P}_{1}}$ and $\Gamma_{\mathscr{P}_{2}}$ are as follows:

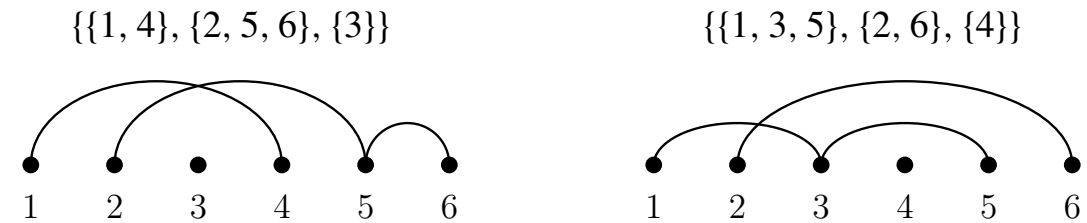

The next proposition shows that in order to compute Betti numbers $\beta_{r}\left(G, K_{n}\right)$ for arbitrary graphs $G$, we need only study the Betti numbers of nonnesting arc diagrams.

Proposition 2.4 [Braun et al. 2011, Proposition 5.6]. For any graph $G$ on vertex set $[\mathrm{m}]$, there exists a unique nonnesting partition $\mathscr{P}$ of $[\mathrm{m}]$ such that $\Gamma_{\mathscr{P}}$ is a subgraph of $G$ and $\operatorname{OHOM}\left(G, K_{n}\right)=\operatorname{OHOM}\left(\Gamma_{\mathscr{P}}, K_{n}\right)$. We call $\Gamma_{\mathscr{P}}$ the reduced arc diagram for $G$.

Suppose there exist vertices $1 \leq a \leq b<c \leq d \leq m$ in $G$ such that $(a, d)$ and $(b, c)$ lie in $E(G)$ (so that the edge $(b, c)$ is nested below the edge $(a, d)$ ), 
and let $G^{\prime}$ be the graph obtained from $G$ by removing the edge $(a, d)$. The proof of Proposition 2.4 uses the observation that $\operatorname{OHOM}\left(G, K_{n}\right)=\operatorname{OHOM}\left(G^{\prime}, K_{n}\right)$ so that the reduced graph $\Gamma_{\mathscr{P}}$ is obtained from $G$ by inductively removing the "top" arc in any pair of nested edges in $G$.

The goal for the remainder of this section is to describe a natural partial order on the family of nonnesting partitions of $[m]$. We then describe how to use this partial order to compute the Betti numbers $\beta_{r}\left(\Gamma_{\mathscr{P}}, K_{n}\right)$ of an arc diagram. For further information on posets and definitions of any undefined terms, we refer the reader to [Stanley 1997].

Definition 2.5. The $m$-th diagram poset, denoted $\mathscr{D}_{m}=\left(\mathscr{D}_{m}, \preceq\right)$, is the poset whose elements are arc diagrams of nonnesting partitions of $[m]$, partially ordered by $\mathscr{P} \preceq \mathscr{Q}$ if every arc of $\mathscr{Q}$ lies above an arc of $\mathscr{P}$.

The minimal element of $\mathscr{D}_{m}$ is the path of length $m-1$ on $[m]$, and the maximal element of $\mathscr{D}_{m}$ is the empty graph.

For example, there are five nonnesting partitions of [3]:

$$
\begin{aligned}
& \mathscr{P}_{1}=\{\{1\},\{2\},\{3\}\}, \\
& \mathscr{P}_{2}=\{\{1,3\},\{2\}\}, \\
& \mathscr{P}_{3}=\{\{1,2\},\{3\}\}, \\
& \mathscr{P}_{4}=\{\{1\},\{2,3\}\}, \\
& \mathscr{P}_{5}=\{\{1,2,3\}\} .
\end{aligned}
$$

Let $\Gamma_{1}, \ldots, \Gamma_{5}$ denote their corresponding arc diagrams, as shown in Figure 1.

If $(P, \leq)$ is a poset, a subset $U \subseteq P$ is a upper order ideal if $y \in U$ whenever $x \in U$ and $y \geq x$. An upper order ideal $U \subseteq P$ is principal if there is an element

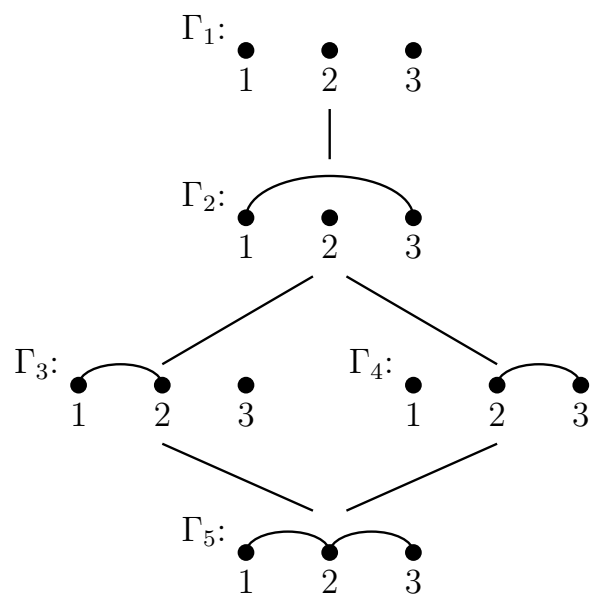

Figure 1. The Hasse diagram for $\mathscr{D}_{3}$. 
$\alpha \in P$ such that $U=\{y \in P: y \geq \alpha\}$. The importance of the partial order on $\mathscr{D}_{m}$ is illustrated in the following proposition.

Proposition 2.6 [Braun et al. 2011, Proposition 5.8]. If $\mathscr{P} \preceq 2$ in $\mathscr{D}_{m}$, then

$$
\mathrm{OHOM}\left(\Gamma_{\mathscr{P}}, K_{n}\right) \subseteq \mathrm{OHOM}\left(\Gamma_{2}, K_{n}\right) .
$$

Further, for each multihomomorphism $\eta \in \mathrm{OHOM}\left(G_{e}, K_{n}\right)$, where $G_{e}$ denotes the empty graph on vertex set $[m]$, the upper order ideal $U(\eta) \subseteq \mathscr{D}_{m}$ of arc diagrams whose OHOM complexes contain $\eta$ is principal.

Proof. Fix a multihomomorphism $\eta \in \operatorname{OHOM}\left(\Gamma_{\mathscr{P}}, K_{n}\right)$. We need to show that each choice $[\varphi(i) \in \eta(i)]_{i=1}^{m}$ yields an order-preserving homomorphism from $\Gamma_{2}$ to $K_{n}$ so that $\eta \in \operatorname{OHOM}\left(\Gamma_{2}, K_{n}\right)$ as well.

Let $(a, d)$ be an edge in $\Gamma_{2}$ with $a<d$. Since $\mathscr{P} \preceq 2$, there is an edge $(b, c)$ in $\Gamma_{\mathscr{P}}$ such that $a \leq b<c \leq d$. Since $\varphi$ is an order-preserving homomorphism from $\Gamma_{\mathscr{P}}$ to $K_{n}$ and $(b, c)$ is an arc in $\Gamma_{\mathscr{P}}$, we see that $\varphi(a) \leq \varphi(b)<\varphi(c) \leq \varphi(d)$. The arc $(a, d)$ was arbitrary, and hence $\varphi(a)<\varphi(d)$ for all arcs $(a, d)$ in $\Gamma_{2}$. Thus $\varphi$ is an order-preserving homomorphism from $\Gamma_{2}$ to $K_{n}$ and $\eta \in \operatorname{OHOM}\left(\Gamma_{2}, K_{n}\right)$, as desired.

Suppose next that $\eta \in \operatorname{OHOM}\left(G_{e}, K_{n}\right)$. Consider the graph $G$ on $[m]$ obtained as the union of all arc diagrams $\Gamma_{2}$ such that $\eta \in \operatorname{OHOM}\left(\Gamma_{2}, K_{n}\right)$, and let $\Gamma_{\mathscr{P}}$ denote the reduced arc diagram of $G$. Clearly $\mathscr{P} \preceq 2$ for all nonnesting partitions 2 whose OHOM complexes contain $\eta$. Thus $U(\eta)$ is generated by $\mathscr{P}$.

Example 2.7. We illustrate Proposition 2.6 for the following multihomomorphism $\eta \in \mathrm{OHOM}\left(\Gamma_{1}, K_{9}\right)$, using the notation from Figure 1:

\begin{tabular}{c|c|c}
$\eta(1)$ & $\eta(2)$ & $\eta(3)$ \\
\hline$x_{1}$ & $x_{4}$ & $x_{7}$ \\
$x_{3}$ & $x_{6}$ & $x_{9}$ \\
& $x_{7}$ &
\end{tabular}

Since $\eta(2) \cap \eta(3)$ is nonempty, the nonnesting partitions $\mathscr{P}$ for which $\eta$ lies in $\operatorname{OHOM}\left(\Gamma_{\mathscr{P}}, K_{9}\right)$ are $\mathscr{P}_{1}, \mathscr{P}_{2}$ and $\mathscr{P}_{3}$. The corresponding graphs $\Gamma_{1}, \Gamma_{2}$, and $\Gamma_{3}$ form an upper order ideal in $\mathscr{D}_{3}$ that is generated by $\Gamma_{3}$.

Weights of nonnesting partition graphs. Proposition 2.6 gives a well defined notion of the minimal arc diagram $\Gamma_{2}$ whose OHOM complex supports a given multihomomorphism $\eta \in \operatorname{OHOM}\left(G_{e}, K_{n}\right)$. We make this more precise in the following definition.

Definition 2.8. Let $\mathscr{P}$ be a nonnesting partition of $[\mathrm{m}]$. The $r$-th weight of $\mathscr{P}$ for $n$, denoted $\omega_{r}(\mathscr{P}, n)$, counts the number of $r$-dimensional multihomomorphisms $\eta \in \operatorname{OHOM}\left(G_{e}, K_{n}\right)$ such that $\mathscr{P}$ generates $U(\eta)$. 
To be more specific, Proposition 2.6 says that for each nonnesting partition 2 and each multihomomorphism $\eta \in \operatorname{OHOM}\left(\Gamma_{2}, K_{n}\right)$, there is a unique minimal nonnesting partition $\mathscr{P} \preceq 2$ such that $\eta \in \operatorname{OHOM}\left(\Gamma_{\mathscr{P}}, K_{n}\right)$. This allows us to partition the $r$-dimensional multihomomorphisms of $\mathrm{OHOM}\left(\Gamma_{2}, K_{n}\right)$ according to the poset $\mathscr{D}_{m}$, as the following proposition indicates.

Proposition 2.9 [Braun et al. 2011, Proposition 5.10]. For any nonnesting partition 2,

$$
\beta_{r}\left(\Gamma_{2}, K_{n}\right)=\sum_{\mathscr{P} \preceq 2} \omega_{r}(\mathscr{P}, n) .
$$

Recall that a collection of vertices $W$ in a graph $G$ is independent if there are no edges in $G$ among the vertices in $W$. The following lemma provides a converse to Proposition 1.4 when computing weights.

Lemma 2.10. Let $\eta$ be a multihomomorphism of $\mathrm{OHOM}\left(G_{e}, K_{n}\right)$, and let $\mathscr{P}$ be the nonnesting partition whose arc diagram generates $U(\eta)$. Suppose $I=[a, c] \subseteq[m]$ is independent in $\Gamma_{\mathscr{P}}$. Then

(1) $\eta(a) \cap \eta(c) \neq \varnothing$,

(2) $|\eta(a) \cap \eta(c)|=1$, and

(3) if $\eta(a) \cap \eta(c)=\left\{x_{i}\right\}$, then $\eta(b)=\left\{x_{i}\right\}$ for all $a<b<c$.

Proof. To prove (1), suppose by way of contradiction that $\eta(a) \cap \eta(c)=\varnothing$. Consider the arc diagram $\Gamma_{2}$ obtained from $\Gamma_{\mathscr{P}}$ by adding the arc $(a, c)$. Since $I$ is independent in $\Gamma_{\mathscr{P}}$, the graph $\Gamma_{\mathscr{2}}$ is the arc diagram of a nonnesting partition 2.

First, we observe that $2 \prec \mathscr{P}$ since $\Gamma_{\mathscr{P}}$ is a subgraph of $\Gamma_{2}$, and hence every arc of $\Gamma_{\mathscr{p}}$ lies above an arc of $\Gamma_{2}$. Next, we claim that $\eta \in \operatorname{OHOM}\left(\Gamma_{2}, K_{n}\right)$. Since $(a, c)$ is the only edge in $E\left(\Gamma_{2}\right) \backslash E\left(\Gamma_{\mathscr{P}}\right)$, we only need to check that $(x, y)$ is an edge of $K_{n}$ for any choice of $x \in \eta(a)$ and $y \in \eta(c)$. This follows immediately from our assumption that $\eta(a) \cap \eta(c)=\varnothing$.

Thus $\eta \in \mathrm{OHOM}\left(\Gamma_{2}, K_{n}\right)$ and $2 \prec \mathscr{P}$, contradicting our assumption that the nonnesting partition $\mathscr{P}$ generates $U(\eta)$. This proves that $\eta(a) \cap \eta(c) \neq \varnothing$. Parts (2) and (3) follow immediately from the requirement that $\eta(a) \leq \eta(b) \leq \eta(c)$ for all $a<b<c$, together with the fact that $\eta(a) \cap \eta(c) \neq \varnothing$.

Lemma 2.11 [Braun et al. 2011, Theorem 5.11]. If $\Gamma_{\mathscr{P}}$ contains an arc $(a, c)$ where $c-a>2$, then $\omega_{r}(\mathscr{P}, n)=0$.

Proof. Suppose to the contrary that $\Gamma_{\mathscr{P}}$ contains such an arc and that $\omega_{r}(\mathscr{P}, n) \neq 0$. Let $\eta$ be an $r$-dimensional multihomomorphism of $\operatorname{OHOM}\left(G_{e}, K_{n}\right)$ such that $\Gamma_{\mathscr{P}}$ generates $U(\eta)$.

Consider the intervals $I=[a, c-1]$ and $I^{\prime}=[a+1, c]$. Since $\mathscr{P}$ is nonnesting, 
$I$ and $I^{\prime}$ are independent in $\Gamma_{\mathscr{P}}$. By Lemma 2.10, there is an element

$$
x_{i} \in \eta(a) \cap \eta(c-1)
$$

and moreover, $\eta(b)=\left\{x_{i}\right\}$ for all $a<b<c-1$. In particular, $\eta(a+1)=\left\{x_{i}\right\}$ since $a+1<c-1$. By applying Lemma 2.10 to the interval $I^{\prime}$, we see that $\eta(a+1) \cap \eta(c) \neq \varnothing$ and hence $x_{i} \in \eta(c)$. Thus $x_{i} \in \eta(a) \cap \eta(c)$, which contradicts Proposition 1.4.

Following [Braun et al. 2011], we call an arc diagram $\Gamma_{\mathscr{P}}$ containing no arcs of the form $(i, j)$ with $j-i>2$ a small arc diagram, and we say that the corresponding nonnesting partition $\mathscr{P}$ is a small nonnesting partition. In light of Lemma 2.11, we need only compute the weights $\omega_{r}\left(\mathscr{P}, K_{n}\right)$ for which $\Gamma_{\mathscr{P}}$ is a small arc diagram. The following two results are interesting enumerative results in their own right.

Proposition 2.12 ([Stanley 1997]). The number of nonnesting arc diagrams on $[m]$ is enumerated by the $m$-th Catalan number

$$
C_{m}=\frac{1}{m+1}\left(\begin{array}{c}
2 m \\
m
\end{array}\right) \text {. }
$$

Proposition 2.13 ([Braun et al. 2011, Theorem 5.12]). Let $F_{m}$ be the $m$-th Fibonacci number with $F_{0}=F_{1}=1$. The number of small arc diagrams on $[\mathrm{m}]$ is $F_{2 m-2}$.

An example. As a more complicated example, we exhibit the weights and corresponding Betti numbers for all nonnesting partitions of $\{1,2,3\}$. We recall the arc diagrams $\Gamma_{1}, \ldots, \Gamma_{5}$ used in Figure 1.

Proposition 2.14. For all $r, n \geq 0$,

$$
\omega_{r}\left(\Gamma_{1}, K_{n}\right)=\left(\begin{array}{c}
n \\
r+1
\end{array}\right)(r+1) .
$$

Proof. Let $\eta \in \mathrm{OHOM}\left(\Gamma_{1}, K_{n}\right)$ be a multihomomorphism whose upper order ideal $U(\eta)$ is generated by $\Gamma_{1}$. By Lemma 2.10, there is a single element $x_{i} \in \eta(1) \cap \eta(3)$ and $\eta(2)=\left\{x_{i}\right\}$. In order to compute $\omega_{r}\left(\Gamma_{1}, K_{n}\right)$, we first determine that there are $r+1$ distinct elements in $\eta(1) \cup \eta(2) \cup \eta(3)$. Indeed, by the inclusion-exclusion principle,

$$
\begin{aligned}
\mid \eta(1) & \cup \eta(2) \cup \eta(3) \mid \\
= & |\eta(1)|+|\eta(2)|+|\eta(3)|-|\eta(1) \cap \eta(2)|-|\eta(1) \cap \eta(3)|-|\eta(2) \cap \eta(3)| \\
& +|\eta(1) \cap \eta(2) \cap \eta(3)| \\
= & (r+3)-3+1=r+1 .
\end{aligned}
$$


In order to describe any such multihomomorphism $\eta$, we must choose a subset $X \subseteq\left\{x_{1}, \ldots, x_{n}\right\}$ of the $r+1$ distinct elements in $\eta(1) \cup \eta(2) \cup \eta(3)$, together with the single element $x_{i} \in X$ that is common to all three sets. Certainly there are $\left(\begin{array}{c}n \\ r+1\end{array}\right)(r+1)$ ways to make these choices. Having chosen $X$ and $x_{i} \in X$, we take

$$
\eta(1)=\left\{x \in X: x \leq x_{i}\right\}, \quad \eta(2)=\left\{x_{i}\right\}, \quad \text { and } \quad \eta(3)=\left\{x \in X: x \geq x_{i}\right\} .
$$

Proposition 2.15. For all $r, n \geq 0$,

$$
\omega_{r}\left(\Gamma_{2}, K_{n}\right)=\left(\begin{array}{c}
n \\
r+1
\end{array}\right)\left(\begin{array}{c}
r+1 \\
2
\end{array}\right) .
$$

Proof. Let $\eta \in \mathrm{OHOM}\left(\Gamma_{2}, K_{n}\right)$ be an $r$-dimensional multihomomorphism whose upper order ideal $U(\eta)$ is generated by $\Gamma_{2}$. By Lemma 2.10, there is an element $x_{i} \in \eta(1) \cap \eta(2)$ and another element $x_{j} \in \eta(2) \cap \eta(3)$. Moreover, by Proposition 1.4, $\eta(1) \cap \eta(3)=\varnothing$ and hence $x_{i} \neq x_{j}$. Thus by the inclusion-exclusion principle, there are $r+1$ distinct elements in $\eta(1) \cup \eta(2) \cup \eta(3)$.

In order to describe any such multihomomorphism $\eta$, we must first choose a subset $X \subseteq\left\{x_{1}, \ldots, x_{n}\right\}$ of the $r+1$ elements in $\eta(1) \cup \eta(2) \cup \eta(3)$, together with the elements $x_{i} \in \eta(1) \cap \eta(2)$ and $x_{j} \in \eta(2) \cap \eta(3)$. Certainly there are $\left(\begin{array}{c}n \\ r+1\end{array}\right)\left(\begin{array}{c}r+1 \\ 2\end{array}\right)$ ways to make these choices. Given the set $X$ and distinguished elements $x_{i}$ and $x_{j}$, we take

$$
\eta(1)=\left\{x \in X: x \leq x_{i}\right\}, \quad \eta(2)=\left\{x \in X: x_{i} \leq x \leq x_{j}\right\}, \quad \eta(3)=\left\{x \in X: x \geq x_{j}\right\} .
$$

Proposition 2.16. For all $r, n \geq 0$,

$$
\omega_{r}\left(\Gamma_{3}, K_{n}\right)=\left(\begin{array}{c}
n \\
r+2
\end{array}\right)\left(\begin{array}{c}
r+2 \\
2
\end{array}\right)
$$

Proof. Let $\eta \in \operatorname{OHOM}\left(\Gamma_{3}, K_{n}\right)$ be an $r$-dimensional multihomomorphism whose upper order ideal $U(\eta)$ is generated by $\Gamma_{3}$. By Lemma 2.10, there is an element $x_{j} \in \eta(2) \cap \eta(3)$, and by Proposition 1.4, $\eta(1) \cap \eta(2)=\varnothing$. By the inclusionexclusion principle, there are $r+2$ distinct elements in $\eta(1) \cup \eta(2) \cup \eta(3)$.

In order to describe any such multihomomorphism $\eta$, we must first choose a subset $X \subseteq\left\{x_{1}, \ldots, x_{n}\right\}$ of the $r+2$ distinct elements in $\eta(1) \cup \eta(2) \cup \eta(3)$, together with the element $x_{j} \in \eta(2) \cap \eta(3)$ and the largest element $x_{i}$ in $\eta(1)$. Certainly there are $\left(\begin{array}{c}n \\ r+2\end{array}\right)\left(\begin{array}{c}r+2 \\ 2\end{array}\right)$ ways to make these choices. As before, having chosen $X, x_{i}$ and $x_{j}$, we take

$$
\eta(1)=\left\{x \in X: x \leq x_{i}\right\}, \quad \eta(2)=\left\{x \in X: x_{i}<x \leq x_{j}\right\}, \quad \eta(3)=\left\{x \in X: x \geq x_{j}\right\} .
$$

Proposition 2.17. For all $r, n \geq 0$,

$$
\omega_{r}\left(\Gamma_{4}, K_{n}\right)=\left(\begin{array}{c}
n \\
r+2
\end{array}\right)\left(\begin{array}{c}
r+2 \\
2
\end{array}\right)
$$


Proof. The proof of this proposition follows by an argument that is symmetric to the one given to compute the weights $\omega_{r}\left(\Gamma_{3}, K_{n}\right)$.

Proposition 2.18. For all $r, n \geq 0$,

$$
\omega_{r}\left(\Gamma_{5}, K_{n}\right)=\left(\begin{array}{c}
n \\
r+3
\end{array}\right)\left(\begin{array}{c}
r+2 \\
2
\end{array}\right) .
$$

Proof. Let $\eta \in \operatorname{OHOM}\left(\Gamma_{5}, K_{n}\right)$ be an $r$-dimensional multihomomorphism whose upper order ideal $U(\eta)$ is generated by $\Gamma_{5}$. By Proposition 1.4, $\eta(1) \cap \eta(2)$, $\eta(2) \cap \eta(3)$, and $\eta(1) \cap \eta(3)$ are empty. Thus by the inclusion-exclusion principle, $|\eta(1) \cup \eta(2) \cup \eta(3)|=r+3$.

In order to describe such a multihomomorphism $\eta$, we must choose a subset $X \subseteq\left\{x_{1}, \ldots, x_{n}\right\}$ of the $r+3$ distinct elements of $\eta(1) \cup \eta(2) \cup \eta(3)$ together with the maximal elements $x_{i}$ and $x_{j}$ of $\eta(1)$ and $\eta(2)$ respectively. Having made these choices, we take

$\eta(1)=\left\{x \in X: x \leq x_{i}\right\}, \quad \eta(2)=\left\{x \in X: x_{i}<x \leq x_{j}\right\}, \quad \eta(3)=\left\{x \in X: x>x_{j}\right\}$.

Since $\eta(3)$ must be nonempty, we cannot choose $x_{j}$ to be the maximal element of $X$. The number of ways to choose $X, x_{i}$, and $x_{j}$ is $\left(\begin{array}{c}n \\ r+3\end{array}\right)\left(\begin{array}{c}r+2 \\ 2\end{array}\right)$, which completes the proof.

\section{Enumerative results}

Our goal for this section is to prove the promised formula computing the weights $\omega_{r}(\mathscr{P}, n)$ for any small nonnesting partition $\mathscr{P}$. Before stating the main theorem, we establish notation that will be used for the remainder of the paper.

Proposition 3.1. For any small nonnesting partition $\mathscr{P}$ of $[\mathrm{m}]$, there is a unique constant $k=k(\mathscr{P})$ and a unique decomposition of $[\mathrm{m}]$ into intervals $I_{1}, \ldots, I_{k}$ satisfying the following conditions.

(P1) $I_{1} \cup \cdots \cup I_{k}=[m]$,

(P2) $I_{1} \leq I_{2} \leq \cdots \leq I_{k}$,

(P3) $\left|I_{j}\right| \geq 2$ for all $j$, and

(P4) each interval $I_{j}$ satisfies exactly one of the following conditions:

(i) $I_{j}$ is a maximal interval (under inclusion) that is independent in $\Gamma_{\mathscr{P}}$.

(ii) $I_{j}=\left\{i_{j}, i_{j+1}\right\}$ and $\left(i_{j}, i_{j+1}\right)$ is an edge of $\Gamma_{\mathscr{P}}$.

Proof. We induct on $m$. The result is clear when $m=2$. When $m \geq 3$, we examine two cases.

If $(1,2)$ is an arc in $\Gamma_{\mathscr{P}}$, let $I_{1}=\{1,2\}$. Inductively, we may decompose the restriction of $\mathscr{P}$ to $[2, m]$ into intervals $I_{2}, \ldots, I_{k}$ satisfying conditions (P1)-(P4). 
On the other hand, if $(1,2)$ is not an $\operatorname{arc}$ in $\Gamma_{\mathscr{P}}$, let $t$ be the largest element of $[m]$ such that $[1, t]$ is independent in $\Gamma_{\mathscr{P}}$. Let $I_{1}=[1, t]$; if $t=m$, we have found the desired decomposition. Otherwise, if $t<m$, the restriction of $\Gamma_{\mathscr{P}}$ to $[t, m]$ is a small arc diagram, and we may inductively decompose the restriction of $\Gamma_{\mathscr{P}}$ to $[t, m]$ into intervals $I_{2}, \ldots, I_{k}$ satisfying conditions (P1)-(P4).

In either of the above cases, we must check that the resulting interval decomposition $[m]=I_{1} \cup \cdots \cup I_{k}$ satisfies conditions (P1)-(P4). Conditions (P1)-(P3) are satisfied by the inductive hypothesis. We must check, however, that if $I_{1}$ and $I_{2}$ are both edgefree as in condition (P4.i), then both are maximal under inclusion. By our construction, $I_{1}=[1, t]$ is maximal. Since $t+1 \notin I_{1}$ and $\mathscr{P}$ is small, either $(t, t+1)$ or $(t-1, t+1)$ is an edge in $\Gamma_{\mathscr{P}}$. If $(t, t+1)$ is an edge in $\Gamma_{\mathscr{P}}$, then $I_{2}=\{t, t+1\}$ satisfies condition (P4.ii). If $(t-1, t+1)$ is an edge in $\Gamma_{\mathscr{P}}$, then $I_{2}$ satisfies condition (P4.i), and $t-1$ cannot be added to $I_{2}$ without violating the independence condition. Thus $I_{2}$ is maximal under inclusion, which completes the proof.

Example 3.2. Consider the small arc diagram $\Gamma_{\mathscr{P}}$ for

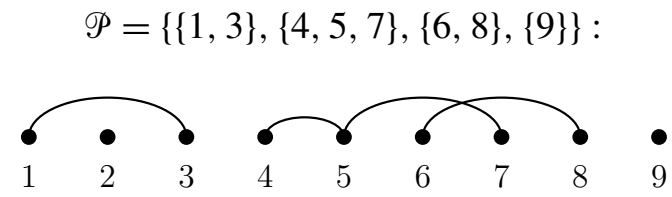

The interval decomposition of $\Gamma_{\mathscr{P}}$ is

$$
\begin{aligned}
& I_{1}=\{1,2\}, \quad I_{2}=\{2,3,4\}, \quad I_{3}=\{4,5\}, \\
& I_{4}=\{5,6\}, \quad I_{5}=\{6,7\}, \quad I_{6}=\{7,8,9\} \text {. }
\end{aligned}
$$

Theorem 3.3. Let $\mathscr{P}$ be a small nonnesting partition of $[\mathrm{m}]$ with interval decomposition $I_{1}, \ldots, I_{k}$ as described by Proposition 3.1. For any $r, n \geq 0$,

$$
\omega_{r}(\mathscr{P}, n)= \begin{cases}\left(\begin{array}{l}
n \\
l
\end{array}\right)\left(\begin{array}{c}
l-1 \\
k
\end{array}\right) & \text { if }(1,2),(m-1, m) \in E\left(\Gamma_{\mathscr{P}}\right) ; \\
\left(\begin{array}{l}
n \\
l
\end{array}\right)\left(\begin{array}{l}
l \\
k
\end{array}\right) & \text { otherwise, }\end{cases}
$$

where $l:=r+m-\sum_{j \in J}\left(\left|I_{j}\right|-1\right)$ and $J \subseteq[k]$ indexes those intervals described by condition (P4.i).

Proof. Fix a small nonnesting partition $\mathscr{P}$ of $[\mathrm{m}]$. For each $1 \leq j \leq k$, let $I_{j}=$ $\left[a_{j}, c_{j}\right]$. For any $r$-dimensional multihomomorphism $\eta \in \operatorname{OHOM}\left(\Gamma_{e}, K_{n}\right)$, we observe that $\sum_{i=1}^{m}|\eta(i)|=r+m$. If the arc diagram for $\Gamma_{\mathscr{P}}$ generates $U(\eta)$, then Lemma 2.10 prescribes the combinatorial structure of the intersections of the sets $\eta(i)$ within each interval $I_{1}, \cdots, I_{k}$. 
As a consequence of these lemmas, we claim that as a set,

$$
l:=|\eta(1) \cup \cdots \cup \eta(m)|=r+m-\sum_{j \in J}\left(\left|I_{j}\right|-1\right),
$$

where $J \subseteq[k]$ indexes those intervals described by condition (P4.i). To see this, we simply observe that for each interval $I_{j}$ with $j \in J$, there is a single element $x_{j}$ common to the sets among $\left\{\eta(p): p \in I_{j}\right\}$. When computing $|\eta(1) \cup \cdots \cup \eta(m)|$, each of these elements $x_{j}$ is overcounted $\left|I_{j}\right|-1$ times.

Thus in order to describe such a multihomomorphism $\eta$, we must first choose a subset $X \subseteq\left\{x_{1}, \ldots, x_{n}\right\}$ of the $l$ distinct elements of $\eta(1) \cup \cdots \cup \eta(m)$. This can be accomplished in $\left(\begin{array}{l}n \\ l\end{array}\right)$ ways.

Now suppose that $(1,2)$ is not an $\operatorname{arc}$ of $\Gamma_{\mathscr{P}}$. The binomial coefficient $\left(\begin{array}{l}l \\ k\end{array}\right)$ counts the number of ways in which we may decompose the set $X$ into pairwise disjoint intervals $A_{0}<A_{1}<\cdots<A_{k}$ so that the sets $A_{1}, \ldots, A_{k}$ are nonempty. This follows from a standard stars-and-bars argument [Stanley 1997, Section 1.2] by arranging the elements of $X$ linearly as

$$
x_{i_{1}} \quad x_{i_{2}} \quad \cdots \quad x_{i_{l-1}} \quad x_{i_{l}},
$$

with $i_{1}<\cdots<i_{l}$ and choosing $k$ of the spaces between consecutive elements of $X$ to partition the set. This includes the possibility of choosing the space to the left of $x_{i_{1}}$, which corresponds to the case that $A_{0}$ is empty.

We now exhibit a bijection between the family of stars-and-bars partitions of $X$ described in the previous paragraph and the collection of multihomomorphisms $\eta \in \operatorname{OHOM}\left(G_{e}, K_{n}\right)$ such that $\eta(1) \cup \cdots \cup \eta(m)=X$ and $\mathscr{P}$ generates $U(\eta)$.

Given pairwise disjoint intervals $A_{0}<A_{1}<\cdots<A_{k}$ that partition $X$ with $A_{1}, \ldots, A_{k}$ nonempty, let $m_{i}$ denote the smallest element of $A_{i}$ for $1 \leq i \leq k$. We determine the sets $\eta(i)$ by declaring that

- $A_{0} \subseteq \eta(1)$,

- $A_{j} \subseteq \eta\left(c_{j}\right)$ for all $1 \leq j \leq k$, and

- $m_{j} \in \eta(b)$ for all $b \in\left[a_{j}, c_{j}\right]$ and all $j \in J$.

Lemma 2.10 and Proposition 1.4 show that this is a bijective correspondence. By symmetry, the same argument applies to the situation that $(m-1, m) \notin \Gamma_{\mathscr{P}}$.

In the case that both $(1,2)$ and $(m-1, m)$ are edges in $\Gamma_{\mathscr{P}}$, an analogous bijection holds, with the exception that $\left(\begin{array}{c}l-1 \\ k\end{array}\right)$ counts the number of partitions of $X$ into nonempty, pairwise disjoint intervals $B_{0}<\cdots<B_{k}$. Here we must require that $B_{0}$ and $B_{k}$ are nonempty, since they describe the elements of $\eta(1)$ and $\eta(m)$, respectively. 
Example 3.4. We illustrate the proof of Theorem 3.3. Let $\mathscr{P}$ be the small partition from Example 3.2. Suppose $l=11$ and (for simplicity) that

$$
\eta(1) \cup \cdots \cup \eta(9)=\left\{x_{1}, \ldots, x_{11}\right\} .
$$

The stars-and-bars decomposition

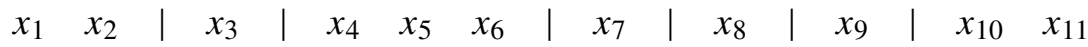

gives

$$
\begin{array}{llll}
A_{0}=\left\{x_{1}, x_{2}\right\}, & A_{1}=\left\{x_{3}\right\}, & A_{2}=\left\{x_{4}, x_{5}, x_{6}\right\}, \quad A_{3}=\left\{x_{7}\right\}, \\
A_{4}=\left\{x_{8}\right\}, & A_{5}=\left\{x_{9}\right\}, & A_{6}=\left\{x_{10}, x_{11}\right\} .
\end{array}
$$

This, in turn corresponds to the following multihomomorphism $\eta$ :

\begin{tabular}{c|c|c|c|c|c|c|c|c}
$\eta(1)$ & $\eta(2)$ & $\eta(3)$ & $\eta(4)$ & $\eta(5)$ & $\eta(6)$ & $\eta(7)$ & $\eta(8)$ & $\eta(9)$ \\
\hline$x_{1}$ & & & & & & & & \\
$x_{2}$ & & & & & & & & \\
$x_{3}$ & $x_{3}$ & & & & & & & \\
& $x_{4}$ & $x_{4}$ & $x_{4}$ & & & & & \\
& & & $x_{5}$ & & & & & \\
& & & $x_{6}$ & & & & & \\
& & & & $x_{7}$ & $x_{8}$ & & & \\
& & & & & $x_{9}$ & $x_{9}$ & & \\
& & & & & & $x_{10}$ & $x_{10}$ & $x_{10}$ \\
& & & & & & & & $x_{11}$
\end{tabular}

We have shaded the blocks $A_{j} \subseteq \eta\left(c_{j}\right)$ for all $1 \leq j \leq 6$, where the intervals $I_{1}, \ldots, I_{6}$ are those given in Example 3.2 and we write $I_{j}=\left[a_{j}, c_{j}\right]$ as in the proof of Theorem 3.3.

\section{Acknowledgments}

We are grateful to Benjamin Braun and Jonathan Browder for a number of insightful conversations and helpful suggestions. Steven Klee's research was supported by NSF VIGRE Grant DMS-0636297.

\section{References}

[Borgs et al. 2006] C. Borgs, J. Chayes, L. Lovász, V. T. Sós, and K. Vesztergombi, "Counting graph homomorphisms", pp. 315-371 in Topics in discrete mathematics, edited by M. Klazar et al., Algorithms Combin. 26, Springer, Berlin, 2006. MR 2007f:05087 Zbl 1129.05050

[Braun et al. 2011] B. Braun, J. Browder, and S. Klee, "Cellular resolutions of ideals defined by simplicial homomorphisms”, preprint, 2011. To appear in Israel J. Math. arXiv 1103.1275 
[Kozlov 2008] D. Kozlov, Combinatorial algebraic topology, Algorithms and Computation in Mathematics 21, Springer, Berlin, 2008. MR 2008j:55001 Zbl 1130.55001

[Reiner 1997] V. Reiner, "Non-crossing partitions for classical reflection groups", Discrete Math. 177:1-3 (1997), 195-222. MR 99f:06005 Zbl 0892.06001

[Stanley 1997] R. P. Stanley, Enumerative combinatorics, vol. 1, Cambridge Studies in Advanced Mathematics 49, Cambridge University Press, Cambridge, 1997. MR 98a:05001 Zbl 0889.05001

Received: 2011-05-27

Imguerra@ucdavis.edu

klee@math.ucdavis.edu
Accepted: 2011-07-11

Mathematical Sciences Building, One Shields Ave., University of California, Davis, CA 95616, United States

Mathematical Sciences Building, One Shields Ave., University of California, Davis, CA 95616, United States http://www.math.ucdavis.edu/ klee/ 
EDITORS

MANAGING EDITOR

Kenneth S. Berenhaut, Wake Forest University, USA, berenhks@wfu.edu

\section{BOARD OF EDITORS}

John V. Baxley Wake Forest University, NC, USA baxley@wfu.edu

Arthur T. Benjamin Harvey Mudd College, USA benjamin@hmc.edu

Martin Bohner Missouri U of Science and Technology, USA bohner@mst.edu

Nigel Boston University of Wisconsin, USA boston@math.wisc.edu

Amarjit S. Budhiraja U of North Carolina, Chapel Hill, USA budhiraj@email.unc.edu

Pietro Cerone Victoria University, Australia pietro.cerone@vu.edu.au

Scott Chapman Sam Houston State University, USA scott.chapman@shsu.edu

Jem N. Corcoran University of Colorado, USA corcoran@colorado.edu

Toka Diagana Howard University, USA tdiagana@howard.edu

Michael Dorff Brigham Young University, USA mdorff@math.byu.edu

Sever S. Dragomir Victoria University, Australia sever@matilda.vu.edu.au

Behrouz Emamizadeh The Petroleum Institute, UAE bemamizadeh@pi.ac.ae

Errin W. Fulp Wake Forest University, USA fulp@wfu.edu

Joseph Gallian University of Minnesota Duluth, USA jgallian@d.umn.edu

Stephan R. Garcia Pomona College, USA stephan.garcia@pomona.edu

Ron Gould Emory University, USA rg@mathcs.emory.edu

Andrew Granville Université Montréal, Canada andrew@dms.umontreal.ca

Jerrold Griggs University of South Carolina, USA griggs@math.sc.edu

Ron Gould Emory University, USA rg@mathcs.emory.edu

Sat Gupta U of North Carolina, Greensboro, USA sngupta@uncg.edu

Jim Haglund University of Pennsylvania, USA jhaglund@math.upenn.edu

Johnny Henderson Baylor University, USA johnny_henderson@baylor.edu

Natalia Hritonenko Prairie View A\&M University, USA nahritonenko@pvamu.edu

Charles R. Johnson College of William and Mary, USA crjohnso@math.wm.edu

Karen Kafadar University of Colorado, USA karen.kafadar@cudenver.edu

K. B. Kulasekera Clemson University, USA kk@ces.clemson.edu

Gerry Ladas University of Rhode Island, USA gladas@math.uri.edu

David Larson Texas A\&M University, USA larson@math.tamu.edu

Suzanne Lenhart University of Tennessee, USA lenhart@math.utk.edu
Chi-Kwong Li

Robert B. Lund

Gaven J. Martin

Mary Meyer

Emil Minchev

Frank Morgan

Mohammad Sal Moslehian

Zuhair Nashed

Ken Ono

Timothy E. O'Brien

Joseph O'Rourke

Yuval Peres

Y.-F. S. Pétermann

Robert J. Plemmons

Carl B. Pomerance

Vadim Ponomarenko

Bjorn Poonen

James Propp

Józeph H. Przytycki

Richard Rebarber

Robert W. Robinson

Filip Saidak

James A. Sellers

Andrew J. Sterge

Ann Trenk

Ravi Vakil

Ram U. Verma

John C. Wierman

Michael E. Zieve
College of William and Mary, USA ckli@math.wm.edu

Clemson University, USA

lund@clemson.edu

Massey University, New Zealand

g.j.martin@massey.ac.nz

Colorado State University, USA

meyer@stat.colostate.edu

Ruse, Bulgaria

eminchev@ hotmail.com

Williams College, USA

frank.morgan@williams.edu

Ferdowsi University of Mashhad, Iran moslehian@ferdowsi.um.ac.ir

University of Central Florida, USA

znashed@mail.ucf.edu

Emory University, USA

ono@mathcs.emory.edu

Loyola University Chicago, USA

tobrie1@luc.edu

Smith College, USA

orourke@cs.smith.edu

Microsoft Research, USA

peres@microsoft.com

Université de Genève, Switzerland petermann@math.unige.ch

Wake Forest University, USA plemmons@wfu.edu

Dartmouth College, USA

carl.pomerance@dartmouth.edu

San Diego State University, USA

vadim@sciences.sdsu.edu

UC Berkeley, USA

poonen@math.berkeley.edu

U Mass Lowell, USA

jpropp@cs.uml.edu

George Washington University, USA

przytyck@gwu.edu

University of Nebraska, USA

rrebarbe@math.unl.edu

University of Georgia, USA

rwr@cs.uga.edu

U of North Carolina, Greensboro, USA

f_saidak@uncg.edu

Penn State University, USA

sellersj@math.psu.edu

Honorary Editor

andy@ajsterge.com

Wellesley College, USA

atrenk@wellesley.edu

Stanford University, USA

vakil@math.stanford.edu

University of Toledo, USA

verma99@msn.com

Johns Hopkins University, USA

wierman@jhu.edu

University of Michigan, USA

zieve@umich.edu

\section{PRODUCTION}

Silvio Levy, Scientific Editor

Sheila Newbery, Senior Production Editor

Cover design: (2008 Alex Scorpan

See inside back cover or http://msp.berkeley.edu/involve for submission instructions.

The subscription price for 2012 is US \$105/year for the electronic version, and \$145/year (+\$35 shipping outside the US) for print and electronic. Subscriptions, requests for back issues from the last three years and changes of subscribers address should be sent to Mathematical Sciences Publishers, Department of Mathematics, University of California, Berkeley, CA 94704-3840, USA.

Involve (ISSN 1944-4184 electronic, 1944-4176 printed) at Mathematical Sciences Publishers, Department of Mathematics, University of California, Berkeley, CA $94720-3840$ is published continuously online. Periodical rate postage paid at Berkeley, CA 94704, and additional mailing offices.

Involve peer review and production are managed by EditFLOW ${ }^{\mathrm{TM}}$ from Mathematical Sciences Publishers.

PUBLISHED BY

mathematical sciences publishers

http://msp.org/

A NON-PROFIT CORPORATION

Typeset in LATEX

Copyright $@ 2012$ by Mathematical Sciences Publishers 


\section{involve 2012 vol. $5 \quad$ no. 1}

Elliptic curves, eta-quotients and hypergeometric functions

DAVID PATHAKJEE, ZEF RosnBRICK AND EUGENE YoONG

Trapping light rays aperiodically with mirrors

ZaChary Mitchell, GRegory Simon and XueYing ZhaO

A generalization of modular forms

ADAM HAQUE

Induced subgraphs of Johnson graphs

RAMIN NAIMI AND JEFFREY SHAW

Multiscale adaptively weighted least squares finite element methods for convection-dominated PDEs

BRIDGET KRAYNIK, YIFEI SUN AND CHAD R. WESTPHAL

Diameter, girth and cut vertices of the graph of equivalence classes of zero-divisors

Blake Allen, Erin Martin, Eric New and Dane Skabelund

Total positivity of a shuffle matrix

Audra McMillan

Betti numbers of order-preserving graph homomorphisms

LAUREN GUERRA AND STEVEN KLEE

Permutation notations for the exceptional Weyl group $F_{4}$

Patricia Cahn, Ruth HaAs, Aloysius G. Helminck, Juan Li and Jeremy

SCHWARTZ

Progress towards counting $D_{5}$ quintic fields

ERIC LARSON AND LARRY ROLEN

On supersingular elliptic curves and hypergeometric functions 